\title{
VEGETATION MAPPING OF SENTINEL-1 AND 2 SATELLITE IMAGES USING CONVOLUTIONAL NEURAL NETWORK AND RANDOM FOREST WITH THE AID OF DUAL-POLARIZED AND OPTICAL VEGETATION INDEXES
}

\author{
N. Ghasemian Sorboni ${ }^{1}$, P. Pahlavani ${ }^{* 1}$, B. Bigdeli ${ }^{2}$ \\ ${ }^{1}$ School of Surveying and Geospatial Engineering, College of Engineering, University of Tehran, Tehran, Iran - (n.ghasemian, \\ pahlavani)@ut.ac.ir \\ ${ }^{2}$ School of Civil Engineering., Shahrood University of Technology, Shahrood, Iran- bigdeli@ shahroodut.ac.ir
}

KEYWORDS: Vegetation mapping, Sentinel-1, Sentinel-2, Random Forest, Convolutional Neural Network

\begin{abstract}
:
Vegetation mapping is one of the most critical challenges of remote sensing society in forestry applications. Sentinel-1 dataset has the potential of vegetation mapping, but because of its limited number of polarizations, full polarized vegetation indexes are not accessible. The Sentinel-2 dataset is more suitable for vegetation mapping because a wide variety of vegetation indexes can be extracted from them. Handling this large number of vegetation indexes needs a robust feature extractor. Convolutional Neural Networks (CNN) extract relevant features through their deep feature layers structure and throw out disturbances from small to large scales. Hence, they can be far useful for classifying remote sensing data when the number of input bands is considerable. After pre-processing Sentinel-1 and 2 datasets and extracting the dual-polarized and optical vegetation indexes, we fed the sentinel-1 vegetation indexes alongside the VV and VH sigma Nought bands to a Random Forest (RF) and 1D CNN classifier. Also, 13 spectral features of the Sentinel-2 and the extracted indexes like Blue Ratio (BR), Vegetation index based on Red Edge (VIRE) and Normalized Near Infrared (NNIR) were imported to a RF and 1D CNN. The classification result of Sentinel-1 data showed that Dual Polarized Soil Vegetation Index (DPSVI) is a good indicator for discriminating vegetation pixels. Also, the experiment on the Sentinel-2 dataset using 1D CNN resulted in True Positive Rate (TPR) and False Positive Rate of 0.839 and 0.034 , respectively.
\end{abstract}

\section{INTRODUCTION}

Using different remote sensing sensors for object extraction is a common issue in classification tasks (Bigdeli, et al. 2015; Bigdeli et al. 2014). Classifying and mapping vegetation is a cardinal issue for managing natural resources because it plays a significant role in influencing global climate change. Also, it contains a broad domain of applications such as designing strategies for the optimization of urban ecosystems, betterconserving plant communities and so on (Xie et al.; 2008, Feng et al., 2015).

Various studies have been done in vegetation mapping; some of them were predictive models based on statistical or machine learning methods (Franklin, 1995). Overall, we can group vegetation mapping methods based on using ground truth reference to supervised and unsupervised methods. Supervised classifiers are widely used since they are more robust than modelbased approaches (Belgiu and Drăguţ, 2016). Recently, Convolutional Neural Networks have been successful in classifying satellite images due to their ability to omit disturbances at different scales (Maggiori et al., 2016; Fu et al., 2017).

The Microwave remote sensing applies the radar signal of longer wavelength $(1 \mathrm{~mm}-1 \mathrm{~m})$ and saves the backscatter energy transformed to Sigma Nought values (Periasamy, 2018). Various studies have applied SAR data to monitor vegetated areas, especially sentinel-1 images (Baumann et al., 2018; Eringery et al., 2018; Ahmed et al., 2018). So, it seems reasonable to use this dataset for mapping vegetation.

Using of spectral information along with radar data can provide more improvement in object classification (Bigdeli and Pahlavani, 2016 and 2017). Due to spectral variability existence in the Sentinel-2 bands, it has more capacity for defining biomass and vegetation indexes. Sentinel-1 images are only available at two polarizations, and this limits the definition of more informative indexes. So, it is necessary to test our vegetation mapping result using sentinel-2 images.

In this paper, we use Sentinel-1 and 2 datasets for masking vegetated pixels. Furthermore, we aim to make a brief comparison of optic and SAR data performance. Also, we examine the performance of the applied classifiers using Receiver Operating Characteristic (ROC) (Fawcett, 2005).

\section{PROPOSED METHOD}

\subsection{Pre-processing}

\subsubsection{Sentinel-1}

Sentinel-1 GRD data are available at dual-polarized, VV, and VH intensity and amplitude bands. It is required to convert the intensity data to Sigma Nought values to extract the relevant vegetation indexes, Dual Pol Diagonal Distance (DPDD), Vertical Dual Depolarization Index (VDDPI) and Dual Pol Soil Vegetation Index (DPSVI). So, calibration is essential. Although GRD data are speckle reduced products, we further reduced the inherent speckle effect by applying a $7 \times 7$ Lee filter. Furthermore, Range Doppler terrain correction was done using SNAP software.

\subsubsection{Sentinel-2}

Sentinel-2 level-1C products are Top Of the Atmosphere (TOA) reflectance values available at 13 spectral bands from visible to shortwave infrared range collected via a single multispectral instrument. For extracting the probably informative indexes, like Green Normalized Difference Vegetation Index (GNDVI), Normalized Vegetation Index (NDVI), Blue Ratio (BR), Green Ratio (GR), it can be advantageous to do atmospheric correction 
by converting the TOA reflectance to the corresponding ground values. Atmospheric correction of sentinel-2 bands was done using QGIS software.

\subsection{Extracting relevant vegetation indexes}

\subsubsection{Sentinel-1}

Several dual-polarized vegetation indexes have been introduced in the literature for biomass and vegetation estimation (Periasamy, 2018). Table 1 illustrates some of the applied indexes.

Table 1: Dual Polarized vegetation indexes

\begin{tabular}{|c|c|}
\hline index & formula \\
\hline $\mathrm{IDPDD}^{1}$ & $\frac{\left(\sigma_{v v(\max )}-\sigma_{v v(i)}\right)+\sigma_{v h(i)}}{\sqrt{2}}$ \\
& \\
\hline $\mathrm{DPDD}^{2}$ & $\frac{\left(\sigma_{v v(i)}+\sigma_{v h(i)}\right)}{\sqrt{2}}$ \\
\hline $\mathrm{VDDPI}^{3}$ & $\frac{\left(\sigma_{v v(i)}+\sigma_{v h(i)}\right)}{\sigma_{v v(i)}}$ \\
\hline $\mathrm{DPSVI}_{\mathrm{i}}{ }^{4}$ & $I D P D D_{(i)} \times V D D P I_{(i)} \times \sigma_{v h(i)}$ \\
\hline $\mathrm{SNBSD}^{5}$ & $\sigma_{v h(i)}-\sigma_{v v(i)}$ \\
\hline
\end{tabular}

\subsubsection{Sentinel-2}

As mentioned before, there are a massive number of indexes beneficial for vegetation mapping in optic bands. Some of the applied indexes in this research have been shown in Table 2 (Muhsoni et al., 2018).

Table 2: Vegetation indexes extracted from Sentinel-2 bands

\begin{tabular}{|c|c|}
\hline Index & Formula \\
\hline BR (blue ratio) & $\left(\frac{R}{B}\right) \times\left(\frac{G}{B}\right) \times\left(\frac{R E_{1}}{B}\right) \times\left(\frac{N I R_{1}}{B}\right)$ \\
\hline $\begin{array}{c}\text { GNDVI (green } \\
\text { normalized } \\
\text { difference } \\
\text { vegetation index) }\end{array}$ & $\frac{N I R_{1}-G}{N I R_{1}+G}$ \\
\hline $\begin{array}{c}\text { SAVI (soil } \\
\text { adjusted } \\
\text { vegetation index) }\end{array}$ & $\frac{(1+0.2) \times\left(N I R_{1}-N I R_{2}\right)}{N I R_{1}+N I R_{2}+0.2}$ \\
\hline $\begin{array}{c}\text { MSAVI(modified } \\
\text { SAVI) }\end{array}$ & $\frac{1}{2}\left[2 \times N I R_{1}+1\right.$ \\
\hline $\begin{array}{c}\text { NDREI } \\
\text { (normalized } \\
\text { difference red } \\
\text { edge index }\end{array}$ & $\frac{N I R_{1}-R E}{N I R_{1}+R E}$ \\
\hline $\begin{array}{c}\text { NDVI } \\
\text { (normalized } \\
\text { difference } \\
\text { vegetation index) }\end{array}$ & $\frac{N I R_{1}-R}{N I R_{1}+R}$ \\
\hline $\begin{array}{c}\text { NDVI2 } \\
\text { NDV }\end{array}$ & $\frac{N I R_{2}-R}{N I R_{2}+R}$ \\
\hline $\begin{array}{c}\text { NDWI } \\
\text { (normalized } \\
\text { difference water } \\
\text { index) }\end{array}$ & $\frac{G-N I R_{1}}{G+N I R_{1}}$ \\
\hline
\end{tabular}

\footnotetext{
${ }^{1}$ Inverse Dual Pol Diagonal Distance

${ }^{2}$ Dual Pol Diagonal Distance

${ }^{3}$ Vertical Dual Depolarization Index
}

\begin{tabular}{|c|c|}
\hline $\begin{array}{c}\text { NNIR } \\
\text { (normalized near } \\
\text { infrared) }\end{array}$ & $\frac{N I R_{1}}{\left(N I R_{1}+R+G\right)}$ \\
\hline $\begin{array}{l}\text { PSRI (plant } \\
\text { senescence } \\
\text { reflectance } \\
\text { index) }\end{array}$ & $\frac{R-B}{R E_{1}}$ \\
\hline $\mathrm{RR}$ (red ratio) & $\left(\frac{N I R_{1}}{R}\right) \times\left(\frac{G}{R}\right) \times\left(\frac{N I R_{1}}{R E_{1}}\right)$ \\
\hline $\begin{array}{c}\text { RVI (ratio } \\
\text { vegetation index) }\end{array}$ & $\frac{N I R_{1}}{R}$ \\
\hline $\begin{array}{c}\text { SVI (sentinel } \\
\text { improved } \\
\text { vegetation index) }\end{array}$ & $\frac{N I R_{2}-R}{N I R_{2}+R}$ \\
\hline $\begin{array}{l}\text { VIRE (vegetation } \\
\text { index based on } \\
\text { red edge) }\end{array}$ & $\frac{10-N I R_{1}}{R E_{1}{ }^{2}}$ \\
\hline $\begin{array}{c}\text { VIRRE } \\
\text { (vegetation index } \\
\text { ratio based on red } \\
\text { edge) }\end{array}$ & $\frac{N I R_{1}}{R E_{1}}$ \\
\hline $\begin{array}{c}\text { WVVI (world } \\
\text { view improved } \\
\text { vegetation index) }\end{array}$ & $\frac{N I R_{2}-R E_{1}}{N I R_{2}+R E_{1}}$ \\
\hline $\begin{array}{l}\text { MTV (modified } \\
\text { triangular } \\
\text { vegetation index) }\end{array}$ & $1.2 \times[1.2 \times(N I R-G)-2.5 \times(R-G)]$ \\
\hline MTV2 (modified & $1.2 \times[1.2 \times(N I R-G)-2.5 \times(R-G)]$ \\
\hline $\begin{array}{l}\text { vegetation index } \\
2 \text { ) }\end{array}$ & $\sqrt{(2 \times N I R+1)^{2}-(6 \times N I R-5 \times \sqrt{R})-0.5}$ \\
\hline $\begin{array}{c}\text { RDVI } \\
\text { (renormalized } \\
\text { difference } \\
\text { vegetation index) }\end{array}$ & {$\left[\frac{(N I R-R)}{(N I R+R)^{2}}\right]$} \\
\hline $\begin{array}{l}\text { VARI } \\
\text { (vegetation } \\
\text { atmospherically } \\
\text { resistant index) }\end{array}$ & $\frac{(G-R)}{(G+R-B)}$ \\
\hline VI green & $\frac{(G-R)}{(G+R)}$ \\
\hline $\begin{array}{l}\text { MSR (modified } \\
\text { simple ratio) }\end{array}$ & $\frac{\left(\frac{N I R}{R}-1\right)}{\sqrt{\left(\frac{N I R}{R}+1\right)}}$ \\
\hline $\begin{array}{c}\text { TVI (triangular } \\
\text { vegetation index) }\end{array}$ & $0.5 \times(120 \times(N I R-G)-200 \times(R-G))$ \\
\hline
\end{tabular}

\subsection{D Convolutional Neural Network}

Some researches applied CNN for fusion of remote sensing data in the aim of classification improvement (Bigdeli et al. 2019).1D Convolutional Neural Networks are one kind of Deep Neural Networks commonly used in 1D signal processing like Time Series analysis. It is somewhat faster than 2D Convolutional Neural Network as it does not require patch generation that can be a demanding work while processing big satellite images with a considerable number of spectral bands. It also can be applied to 2D signals by vectorizing input image and considering each spectrum as an input to the network. Figure 1 shows the architecture of the 1D CNN applied for vegetation mapping in Sentinel-1 and Sentinel-2 data sets. We used different architectures for Sentinel-1 and 2 because of the different number of input features. For Sentinel-1 data set, the number of input

\footnotetext{
${ }^{4}$ Dual Pol Soil Vegetation Index

${ }^{5}$ Sigma Nought Back Scatter Difference
} 
features was 7, including the features mentioned in Table 1 and sigma Nought backscatter values of bands VV and VH. Sentinel2 input features included the vegetation indexes mentioned in Table 2 and the 13 spectral bands of Sentinel-2 data set. So, overall, we imported 36 features to CNN, as illustrated in Figure $1 \mathrm{~b}$.

Figure 1a shows the 1D CNN architecture for Sentinel-1 data set. Each pixel was considered as a $7 \times 1$ spectrum. Fifty spatial filters with size $3 \times 1$ were considered in the first layer. The coefficients of these filters were learned during training. After applying 1D convolution to the input spectrum (pixel), the size of the input was reduced to $5 \times 1$. So, we had 50 features with $5 \times 1$ dimension as the output of the first layer. Next, the Dropout layer was considered to switch off some of the windows of the spatial filters during training. Also, as the third layer, we set a $2 \times 1$ Max Pooling layer to reduce the distortions and disturbances in the data set. After applying Max Pooling with size 2, size of the input was halved. Then the output of the Max Pooling layer was imported to a Batch Normalization layer to make data zero centered and with unit variance. The inputs to the $1 \mathrm{D} \mathrm{CNN}$ are twodimensional. The first dimension (the second number in the parentheses) refers to the width or height and the second one (the third number in the parentheses refers to the number of channels or bands). None values in the parentheses denote the batch size can be any desired number.

Before importing data into the fully connected layers, we need a flattening layer to convert two-dimensional data into one dimension. After applying the flattening layer, $2 \times 50$ vectors were converted to $100 \times 1$. Two fully connected layers were used to partition the extracted feature space to non-vegetated and vegetated areas (dense-1 and dense-2 in figure 1a). Within convolutional and Max Pooling layers, we embedded Dropout and Batch Normalization layers to improve the generalization ability of the network. Figure $1 \mathrm{~b}$ can be explained in the same manner as figure 1a. Please note that in the last layer of figure 1a, there are three output neurons for Sentinel-1 corresponding to the non-vegetate, vegetated, and other classes. Also, in the last layer of figure $1 \mathrm{~b}$, two neurons are corresponding to the vegetated and non-vegetated classes in Sentinel-2 classification results. It is worth noting that the non-linearity ReLU was applied after convolution layers, and negative values were converted to zero. For implementing CNN and RF classifiers, we used the Keras library and Scikit-learn library of python respectively.

\section{RESULTS AND DISCUSSIONS}

\subsection{Ground Truth Data}

Accuracy validation of the applied classification models is necessary. As a result, we selected Ground Truth data using Google Earth Pro software. The main scope of this paper was detecting vegetated pixels. So, just two classes were considered for classification, non-vegetated areas containing water bodies, agricultural land and urban and vegetated areas containing human-made or natural vegetation. Figure 2 shows the distribution of selected polygons on the RGB image. Black polygons are non-vegetated class Ground Truth, and green polygons are vegetated class Ground Truth. We selected almost 3000 samples from non-vegetated and vegetated Ground Truths randomly. So, in total, we considered nearly 6000 samples as Ground Truth. We portioned this data to Train and Test. 70\% of Ground Truth data were set aside as training and the remaining as a test.

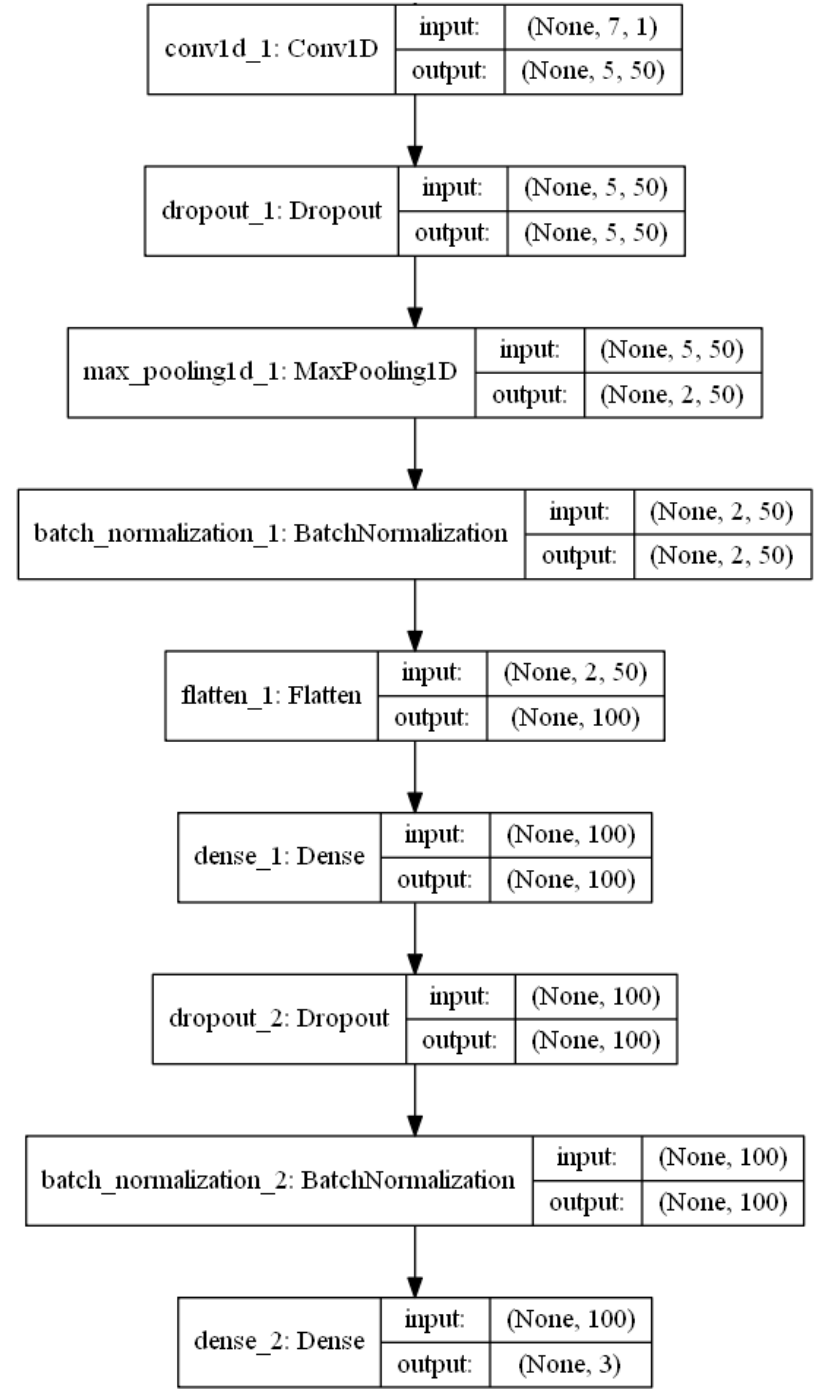

(a) 


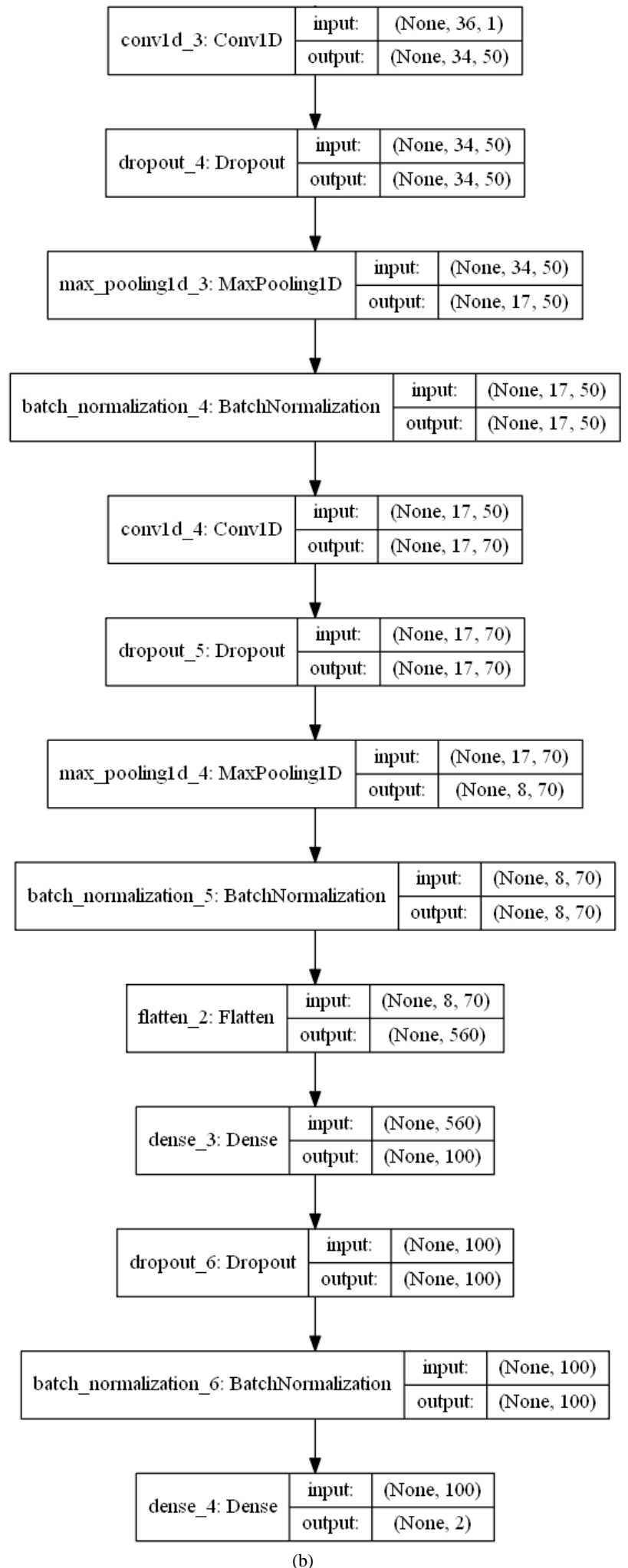

Figure 1: The architecture of the applied 1D CNN; a:Sentinel$1 ; \mathrm{b}:$ Sentinel-2

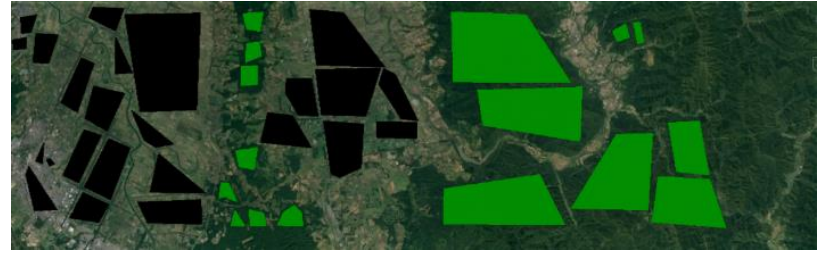

Figure 2: Distribution of Ground Truth Data on the RGB image

\subsection{Masking vegetated pixels using 1-D CNN}

For training proposed 1-D CNN model, for both Sentinel-1 and Sentinel-2 data sets, we applied the same parameters as follows: learning rate parameter must be set a small value to stochastic gradient descent algorithm to work appropriately. So, we set this value to 10-4. Adam algorithm was considered as the optimization method because it usually shows superior performance than other optimization algorithms such as stochastic gradient descent. Also, batch size and the number of epochs parameters were set 100 and 200, respectively. The binary cross-entropy was considered as the loss function, and the weights and biases were updated using the mini-batch method during 200 epochs.

\subsection{Masking vegetated pixels using RF}

Random Forest (RF) classifier is a powerful tool using the concept of randomness and repetition of classifiers. For masking vegetated pixels in both Sentinel-1 and Sentinel-2 data sets, we set the number of trees to 100 and the default value of the selected number of features at each node (the second root of the total number of features) was considered.

Figure 3 shows ROC space for Sentinel-1 RF classifier, the classifier with a combination of features, VV, VH, and DPSVI, achieved the highest TPR because of adding DPSVI that is a good indicator for masking vegetation pixels. The most conservative classifier was the one with a combination of features, $\mathrm{VV}, \mathrm{VH}$, DPDD, and VDDPI, acquired FPR of 0.35. Overall, no significant difference was observed between the performances of the classifiers.

Figure 4 shows the precision and recall of different combinations of features. We can see that while there is not any significant difference between precision values, recall values show different results. Combining DPDD and backscatter values of VV and VH resulted in the lowest recall rate among other combinations. DPDD index inserted uncertainty discriminating water bodies and vegetated pixels because both of them have high diagonal distance in the scatter plot of the Sigma Nought VH and VV bands. When we added DPSVI to the previously mentioned backscatters, highest recall value was acquired because this index is an excellent representative of vegetated areas (Periasamy, 2018). 


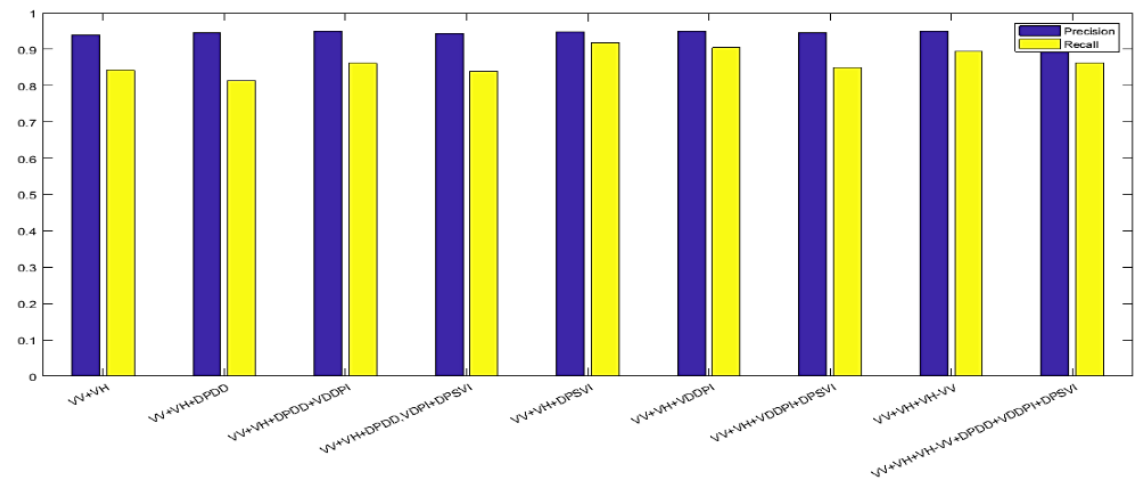

Figure 4: Precision and recall values of different combination of features

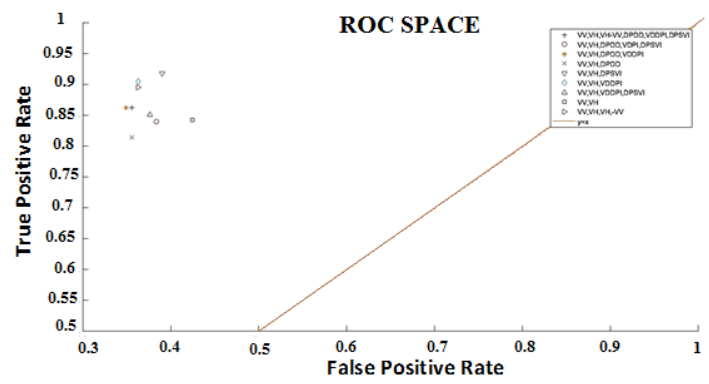

Figure 3: ROC SPACE for different combination of features in Sentinel-1

\section{COMPARISON OF SENTINEL-1 AND SENTINEL-2}

In this section, we aim to compare vegetation detection using Sentinel-1 and Sentinel-2 data. For vegetation detection, we applied both RF and CNN classifiers. We compare the vegetation detection of both data sets in terms of visual inspection and quantitative results.

\subsection{Visual Comparison}

Figure 5 shows the classification results of Senitnel-1 and Sentinel-2 data sets using CNN and RF classifiers. By comparing the classification results with the RGB image we can conclude that using optical bands and their corresponding

Vegetated indexes resulted in the best classification result. RF classifier overestimated in detecting vegetated pixels.

\subsection{Quantitative Comparison}

Figure 6 shows the confusion matrices of the four classification results shown in figure 5. The right part of the Sentinel-1 image was removed after doing Range Doppler Terrain Correction during the pre-processing stage. "Other" Class in the confusion matrices of the Sentinel-1 data set is referring to this part. Classification using RF resulted in 67 and 23 non-vegetated pixels mislabelled as vegetated in Sentinel-2 and Sentinel-1 respectively. Most of these wrongly labelled pixels are related to the agricultural areas located on the west side of the vegetated areas. Applying 1D CNN alongside the optical bands and vegetated indexes resulted in most diagonal confusion matrix and labelled most of the agricultural lands as non-vegetated.
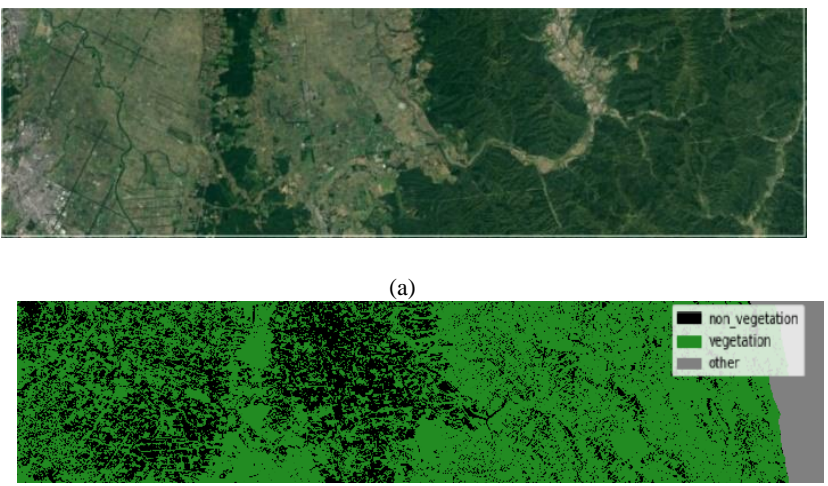

(b)
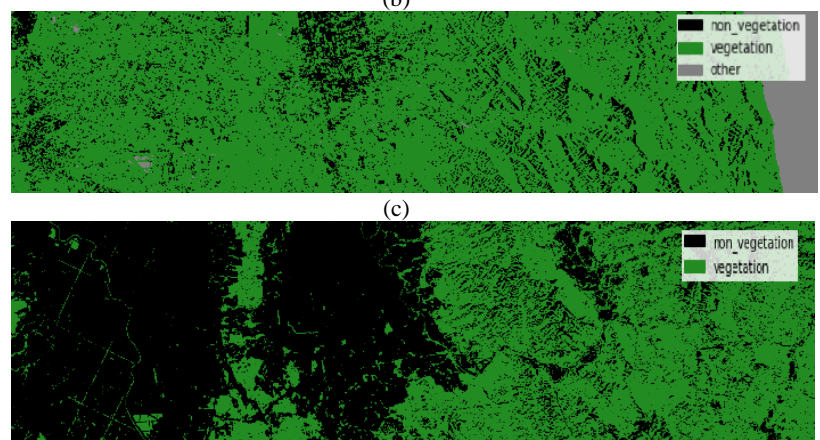

(d)

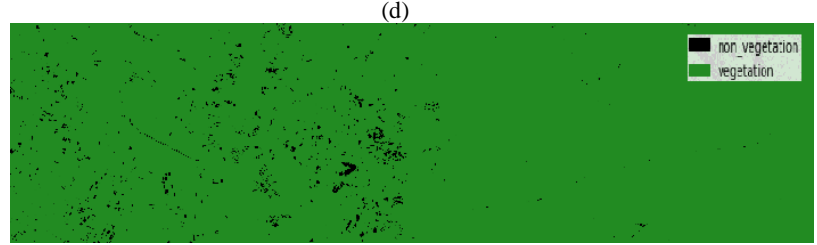

(e)

Figure 5: Classification results on Sentinel-1 and Sentinel-2 data sets; a: RGB image; b: 1D CNN Sentinel-1 data set; c: RF Sentinel-1 data set; d: 1D CNN Sentinel-2 data set; e: RF Sentinel-2 data set.

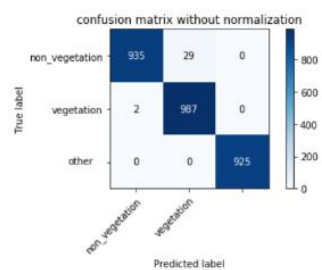

(a)

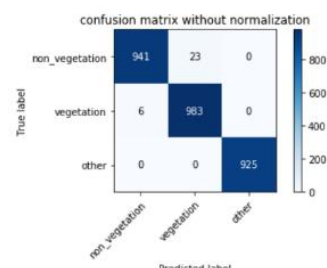

(b) 


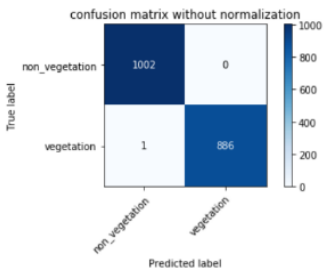

(c)

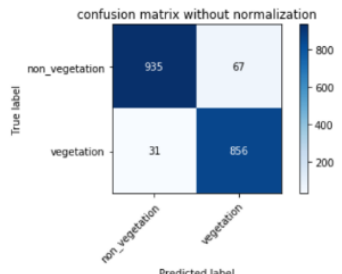

(d)
Figure 6: Confusion matrices of classification results; a: 1D CNN Sentinel-1; b: RF Sentinel-1; c: 1D CNN sentinel-2; d: RF Sentinel-2

Figure 7 shows the ROC curves of our classifications. ROC curves for Sentinel-1 are not very representative of their corresponding classification performances. The least Area Under Curve (AUC) is related to the Sentinel-2 RF classifier because of the large number of negative pixels labelled as positive (vegetated) in figure 5 part e.

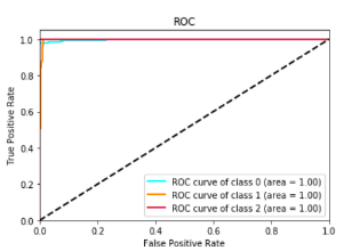

(a)

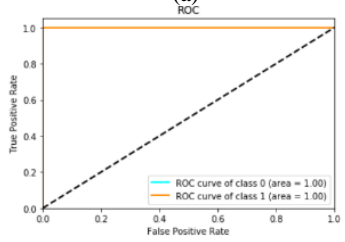

(c)

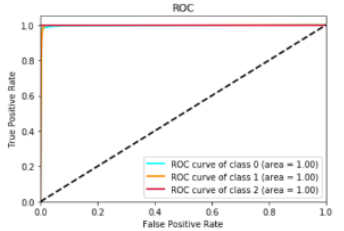

(b)

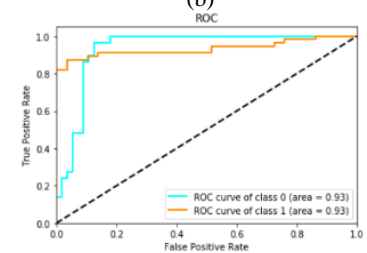

(d)
Figure 7: ROC curves for classification results shown in figure 5

\section{REFERENCES}

Ahmed, A.A., Pradhan, B., Sameen, M.I. and Makky, A.M., 2018. An optimized object-based analysis for vegetation mapping using integration of Quickbird and Sentinel-1 data. Arabian Journal of Geosciences, 11(11), p.280.

Baumann, M., Levers, C., Macchi, L., Bluhm, H., Waske, B., Gasparri, N.I. and Kuemmerle, T., 2018. Mapping continuous fields of tree and shrub cover across the Gran Chaco using Landsat 8 and Sentinel-1 data. Remote sensing of environment, 216, pp.201-211.

Belgiu, M. and Drăguţ, L., 2016. Random forest in remote sensing: A review of applications and future directions. ISPRS Journal of Photogrammetry and Remote Sensing, 114, pp.24-31.

Bigdeli, B., Samadzadegan, F., \& Reinartz, P., 2015. Fusion of hyperspectral and LIDAR data using decision template-based fuzzy multiple classifier system. International Journal of Applied Earth Observation and Geoinformation, 38, 309-320.

Bigdeli, B., Samadzadegan, F., \& Reinartz, P., 2014. Feature grouping-based multiple fuzzy classifier system for fusion of hyperspectral and LIDAR data. Journal of Applied Remote Sensing, 8(1), 083509.
Bigdeli, B., Pahlavani, P., 2016. High resolution multisensor fusion of SAR, optical and LiDAR data based on crisp vs. fuzzy and feature vs. decision ensemble systems, International journal of applied earth observation and geoinformation 52(1): 126-136

Bigdeli, B., Pahlavani, P., 2017. Quad-polarized synthetic aperture radar and multispectral data classification using classification and regression tree and support vector machinebased data fusion system. Journal of applied remote sensing 11(1): 016007

Bigdeli, B., Amini Amirkolaee, H., Pahlavani, P., 2019. Deep feature learning versus shallow feature learning systems for joint use of airborne thermal hyperspectral and visible remote sensing data. International Journal of Remote Sensing, 40(18), 70487070 .

Erinjery, J.J., Singh, M. and Kent, R., 2018. Mapping and assessment of vegetation types in the tropical rainforests of the Western Ghats using multispectral Sentinel-2 and SAR Sentinel1 satellite imagery. Remote sensing of environment, 216, pp.345354.

Fawcett, T., 2006. An introduction to ROC analysis. Pattern recognition letters, 27(8), pp.861-874.

Feng, Q., Liu, J. and Gong, J., 2015. UAV remote sensing for urban vegetation mapping using random forest and texture analysis. Remote Sensing, 7(1), pp.1074-1094.

Franklin, J., 1995. Predictive vegetation mapping: geographic modelling of biospatial patterns in relation to environmental gradients. Progress in physical geography, 19(4), pp.474-499.

Fu, G., Liu, C., Zhou, R., Sun, T. and Zhang, Q., 2017. Classification for high resolution remote sensing imagery using a fully convolutional network. Remote Sensing, 9(5), p.498.

Periasamy, S., 2018. Significance of dual polarimetric synthetic aperture radar in biomass retrieval: An attempt on Sentinel-1. Remote Sensing of Environment, 217, pp.537-549.

Xie, Y., Sha, Z. and Yu, M., 2008. Remote sensing imagery in vegetation mapping: a review. Journal of plant ecology, l(1), pp.9-23.

Lennert, M. and GRASS Development Team, 2017. Addon i.segment.stats. Geographic Resources Analysis Support System (GRASS) Software, Version 7.2, Open Source Geospatial Foundation. grass.osgeo.org/grass $7 /$ manuals/addons/i.segm ent.stats (1 June 2017).

Maas, A., Rottensteiner, F., Heipke, C., 2017. Classification under label noise using outdated maps. ISPRS Ann. Photogramm. Remote Sens. Spatial Inf. Sci., IV-1/W1, 215222. doi.org/10.5194/isprs-annals-IV-1-W1-215-2017.

Maggiori, E., Tarabalka, Y., Charpiat, G. and Alliez, P., 2016, July. Fully convolutional neural networks for remote sensing image classification. In 2016 IEEE International Geoscience and Remote Sensing Symposium (IGARSS) (pp. 5071-5074). IEEE.

Muhsoni, F.F., Sambah34, A., Mahmudi, M. and Wiadnya, D., 2018. Comparison of different vegetation indices for assessing mangrove density using sentinel-2 imagery. Int. J. Geomate, 14, pp.42-51. 